\title{
A NOTE ON THE INVERSE LAPLACE TRANSFORM *
}

\author{
E. Contharteze Grigoletto ${ }^{\dagger}$ E. Capelas de Oliveira ${ }^{\ddagger}$
}

\begin{abstract}
In this note we show that the method for finding inverse Laplace transform without using integration on the complex plane can be used to find integral representations on the positive real axis for some functions. As application of this procedure, some integral representations are obtained.
\end{abstract}

\section{Introduction}

It is known that the Laplace transform theory is an efficient and sophisticated way to solve, among other things, linear differential equations (ordinary and partial) specifically those with constant coefficients. In specific problems, the Laplace transform leads the starting problem (differential equation and initial conditions) to the so-called auxiliary problem. So, our starting problem is converted into another one of seemingly simpler solution. If we can evaluate the inverse Laplace transform, solution of the auxiliary problem, we recover the solution of the starting problem [1, 2, 3, 4, The problem then becomes finding the inverse Laplace transform [5].

The Laplace inversion is generally done numerically due to the impossibility of the exact inversion by means of an integration on the complex plane [6, 7]. When possible, we can make use of the table of Laplace transforms. In the study of luminescence decay of inorganic solids, M. N. Berberan-Santos [8] proposed a method to evaluate the inverse Laplace transform without using integration on the complex plane.

The main objective of this work is primarily to shown some applications of the technique for computing the inverse Laplace transform proposed by M. N. Berberan-Santos, for the graduate students and researchers, who are interested not only in learning about the Laplace transform tool, but also in learning about different techniques used in the theory [9].

The note is organized as follows: Section 1 presents a review involving the Laplace transform and its inverse; The Theorem 2.1, as a review of evaluation of inverse Laplace transforms without the aid of contour integration is shown in Section 2 Section 3 presents, as application, examples in connection with the theory developed in the previous sections. Concluding remarks close the note.

\footnotetext{
Key words: Laplace transform, Inverse Laplace transform, Integral representations

${ }^{\dagger}$ Department of Bioprocess and Biotechnology, DBB/FCA-UNESP, eliana.contharteze@unesp.br

${ }^{\ddagger}$ Department of Applied Mathematics, IMECC/UNICAMP, capelas@ime.unicamp.br
} 


\section{Laplace transform and its inverse}

In this section we review the definition of the Laplace transform and its inverse and we use an illustrative example for computing the inverse Laplace transform by using the complex inversion formula.

Let $\mathcal{S}(\mathbb{R})$ denote the Schwartz space of functions $\}^{1} f: \mathbb{R} \rightarrow \mathbb{C}$ such that $f \in \mathrm{C}^{\infty}(\mathbb{R})$, i.e., $f$ is infinitely differentiable on $\mathbb{R}$, and for all integers $m, n \geq 0$,

$$
\sup _{x \in \mathbb{R}}\left|x^{m} f^{(n)}(x)\right|<\infty,
$$

where sup denotes the supremum. Thus $f \in \mathcal{S}(\mathbb{R})$ means that $f$ is a smooth rapidly decreasing function 10, 11. For example, the function $f(x)=\mathrm{e}^{-x^{2}}$ is such that $f \in \mathcal{S}(\mathbb{R})$ because $f \in \mathrm{C}^{\infty}(\mathbb{R})$ and $\lim _{|x| \rightarrow \infty} x^{m} f^{(n)}(x)=0$, for all integers $m, n \geq 0$, imply $\sup _{x \in \mathbb{R}}\left|x^{m} f^{(n)}(x)\right|<\infty$.

Let $f(t)$ be a real function of (time) variable $t \geq 0$. The Laplace transform of $f$, denoted by $\mathscr{L}[f](s)=\mathrm{F}(s)$, is defined as follows:

$$
\mathscr{L}[f](s)=\mathrm{F}(s)=\int_{0}^{\infty} e^{-s t} f(t) \mathrm{d} t
$$

whenever the integral converges for ${ }^{2} \mathcal{R}[s] \geq \sigma>0$, where $s=\sigma+i \tau$, with $\sigma$ and $\tau$ real numbers, and $\mathrm{F}(s)=0$ for $\sigma<0$.

The inverse Laplace transform of $\mathrm{F}(s)$ is given by the formula

$$
\mathscr{L}^{-1}[\mathrm{~F}](t)=f(t)=\frac{1}{2 \pi i} \lim _{\tau \rightarrow \infty} \int_{\sigma-i \tau}^{\sigma+i \tau} e^{s t} \mathrm{~F}(s) \mathrm{d} s,
$$

where $\sigma$ is large enough that $\mathrm{F}(s)$ is defined for $\mathcal{R}[s] \geq \sigma>0$.

The direct and inverse Laplace transforms are inverse to each other for functions $f \in \mathcal{S}(\mathbb{R})$.

Definition 1.1. (Holomorphic function) The function $\mathrm{F}: \mathcal{U} \rightarrow \mathbb{C}$, where $\mathcal{U}$ is an open subset of $\mathbb{C}$, is holomorphic at $\mathrm{s}$ if

$$
\mathrm{F}^{\prime}(s)=\lim _{h \rightarrow 0} \frac{\mathrm{F}(s+h)-\mathrm{F}(s)}{h}
$$

exists for $s \in \mathbb{C}$.

Theorem 1.1 (Cauchy's Residue Theorem). Let $G(s)$ be holomorphic function on a region $\mathcal{D}$ except for isolated singularities (poles) at $s_{1}, \ldots, s_{n} \in \mathcal{D}$. Let $C$ be any contour curve in $\mathcal{D}$, then

$$
\oint_{C} G(s) \mathrm{d} s=2 \pi i \sum_{k=1}^{n} \operatorname{Res}\left(G ; s_{k}\right)
$$

where

$$
\operatorname{Res}\left(G ; s_{k}\right)=\frac{1}{(m-1) !} \lim _{s \rightarrow s_{k}}\left\{\frac{\mathrm{d}^{\mathrm{m}-1}}{\mathrm{ds}^{m-1}}\left[\left(s-s_{k}\right)^{m} G(s)\right]\right\},
$$

if $m$ is the order of the pole $s_{k}$.

\footnotetext{
${ }^{1} \mathbb{C}=\{a+b i: a, b \in \mathbb{R}\}$.

${ }^{2} \mathcal{R}[s]$ indicates the real part of $s$ and the imaginary part is denoted by $\mathcal{I}[s]$.
} 
Proof of Theorem 1.1. See [6].

If the singularities are not poles, equation (1.4) cannot be used [12].

Let us sketch an explicit calculation involving the inverse Laplace transform.

Example 1.1. Find $\mathscr{L}^{-1}\left[\frac{1}{s^{2}+1}\right]$.

The complex function $\mathrm{F}(s)=\frac{1}{s^{2}+1}$ has poles at $s=i$ and $s=-i$. In this case, the Bromwich contour is shown in Figure 1

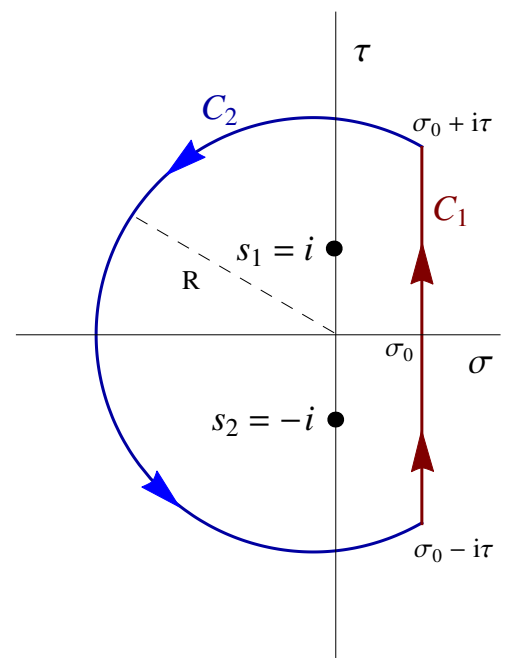

Figure 1: Bromwich contour of $\mathrm{F}(\mathrm{s})$.

Using equation (1.2), the inverse Laplace transform is given by

$$
f(t)=\frac{1}{2 \pi i} \lim _{\tau \rightarrow \infty} \int_{\sigma-i \tau}^{\sigma+i \tau} \frac{e^{s t}}{s^{2}+1} \mathrm{~d} s=\frac{1}{2 \pi i} \lim _{\mathrm{R} \rightarrow \infty} \oint_{\mathrm{C}} \frac{e^{s t}}{s^{2}+1} \mathrm{~d} s,
$$

where $\mathcal{R}[s]=\sigma>0$. We note that the function

$$
\mathrm{G}(s)=\frac{e^{s t}}{s^{2}+1}
$$

which appears in equation $(1.5)$, is holomorphic in the region delimited by the curve $\mathrm{C}=\mathrm{C}_{1} \cup \mathrm{C}_{2}$, where $\mathrm{C}_{1}$ is the vertical segment and $\mathrm{C}_{2}$ is the portion of the circle with radius $\mathrm{R}$ centered at the origin as shown in Figure 1, except at isolated singularities $s_{1}=i$ and $s_{2}=-i$. By Theorem 1.1, equation (1.5) can be rewritten in the following form

$$
f(t)=\frac{1}{2 \pi i} \lim _{\mathrm{R} \rightarrow \infty} \oint_{\mathrm{C}} \frac{e^{s t}}{s^{2}+1} \mathrm{~d} s=\frac{1}{2 \pi i} 2 \pi i \sum_{k=1}^{2} \operatorname{Res}\left(\mathrm{G} ; s_{k}\right)=\sum_{k=1}^{2} \operatorname{Res}\left(\mathrm{G} ; s_{k}\right) .
$$

Function $\mathrm{G}$ has two simple poles $(m=1)$ at $s_{1}=i$ and $s_{2}=-i$. The residues are given by

$$
\operatorname{Res}(\mathrm{G} ; i)=\lim _{s \rightarrow i} \frac{(s-i) e^{s t}}{s^{2}+1}=\frac{e^{i t}}{2 i}
$$


and

$$
\operatorname{Res}(\mathrm{G} ;-i)=\lim _{s \rightarrow-i} \frac{(s+i) e^{s t}}{s^{2}+1}=-\frac{e^{-i t}}{2 i} .
$$

Substituting equations 1.7 and 1.8 into equation 1.6 , we obtain

$$
f(t)=\frac{e^{i t}-e^{-i t}}{2 i}=\sin t
$$

\section{Laplace transform without using integration on the complex plane}

In this section we present, through the next theorem, the inverse Laplace transform without integration on the complex plane as proposed by M. N. Berberan-Santos [8].

Theorem 2.1. If the complex function $F(s)$ is analytic for $\mathcal{R}[s] \geq \sigma>0$ and represents the Laplace transform of a function $f: \mathbb{R} \rightarrow \mathbb{R}$, with $f(t)=0$ for $t<0$, belonging to the space $\mathcal{S}(\mathbb{R})$, then for $t>0$ the expression

$$
f(t)=\frac{e^{\sigma t}}{\pi} \int_{0}^{\infty}\{\mathcal{R}[F(\sigma+i \tau)] \cos (t \tau)-\mathcal{I}[F(\sigma+i \tau)] \sin (t \tau)\} \mathrm{d} \tau
$$

for any real number $\sigma$, provides the real function $f$ whose Laplace transform is $F(s)$.

Proof of Theorem 2.1. Writing $s=\sigma+i \tau$ in equation (1.1) and considering $f(t)=0$ for $t<0$, we can write

$$
\mathrm{F}(\sigma+i \tau)=\int_{-\infty}^{\infty} e^{-\sigma t} f(t) e^{-i \tau t} \mathrm{~d} t
$$

We know that $\mathrm{F}(\sigma+i \tau)$ in equation 2.10 represents the Fourier transform of the function $\varphi(t)=$ $e^{-\sigma t} f(t)$. Then, with the conditions imposed on $f$, there exists an $\sigma>0$ such that for $\mathcal{R}[s]=\sigma$ we may evaluate the inverse Fourier transform of $\varphi(t)$ given by

$$
\mathrm{e}^{-\sigma t} f(t)=\frac{1}{2 \pi} \int_{-\infty}^{\infty} \mathrm{F}(\sigma+i \tau) e^{i \tau t} \mathrm{~d} \tau .
$$

On the other hand, equation 2.11 can be rewritten as

$$
f(t)=\frac{e^{\sigma t}}{2 \pi} \int_{-\infty}^{\infty} e^{i t \tau} \mathrm{F}(\sigma+i \tau) \mathrm{d} \tau
$$

Separating the real and imaginary parts of $\mathrm{F}(\sigma+i \tau)$ in equation 2.12 , we can express

$$
\mathrm{F}(\sigma+i \tau)=\mathcal{R}[\mathrm{F}(\sigma+i \tau)]+i \mathcal{I}[\mathrm{F}(\sigma+i \tau)]
$$

and using the Euler formula $e^{i t \tau}=\cos (t \tau)+i \sin (t \tau)$, we have the following expression

$$
\begin{array}{r}
f(t)=\frac{e^{\sigma t}}{2 \pi}\left\{\int_{-\infty}^{\infty}[\mathcal{R}[\mathrm{F}(\sigma+i \tau)] \cos (t \tau)-\mathcal{I}[\mathrm{F}(\sigma+i \tau)] \sin (t \tau)] \mathrm{d} \tau+\right. \\
\left.+i \int_{-\infty}^{\infty}[\mathcal{I}[\mathrm{F}(\sigma+i \tau)] \cos (t \tau)+\mathcal{R}[\mathrm{F}(\sigma+i \tau)] \sin (t \tau)] \mathrm{d} \tau\right\} .
\end{array}
$$


Since $f(t)$ is a real function, we can write

$$
\int_{-\infty}^{\infty}[\mathcal{I}[\mathrm{F}(\sigma+i \tau)] \cos (t \tau)+\mathcal{R}[\mathrm{F}(\sigma+i \tau)] \sin (t \tau)] \mathrm{d} \tau=0
$$

We thus derive the following expression for $f(t)$ :

$$
f(t)=\frac{e^{\sigma t}}{2 \pi} \int_{-\infty}^{\infty}[\mathcal{R}[\mathrm{F}(\sigma+i \tau)] \cos (t \tau)-\mathcal{I}[\mathrm{F}(\sigma+i \tau)] \sin (t \tau)] \mathrm{d} \tau .
$$

Making the substitution $s=\sigma+i \tau$ and using the Euler formula in equation (1.1), we obtain the relations

and

$$
\mathcal{R}[\mathrm{F}(\sigma+i \tau)]=\int_{0}^{\infty} e^{-\sigma t} f(t) \cos (t \tau) \mathrm{d} t
$$

$$
\mathcal{I}[\mathrm{F}(\sigma+i \tau)]=-\int_{0}^{\infty} e^{-\sigma t} f(t) \sin (t \tau) \mathrm{d} t
$$

Then, by means of equations 2.14 and 2.15 , we note that the function

$$
\psi(\tau)=[\mathcal{R}[\mathrm{F}(\sigma+i \tau)] \cos (t \tau)-\mathcal{I}[\mathrm{F}(\sigma+i \tau)] \sin (t \tau)]
$$

has even parity, then equation 2.13 can be rewritten as

$$
f(t)=\frac{e^{\sigma t}}{\pi} \int_{0}^{\infty}[\mathcal{R}[\mathrm{F}(\sigma+i \tau)] \cos (t \tau)-\mathcal{I}[\mathrm{F}(\sigma+i \tau)] \sin (t \tau)] \mathrm{d} \tau,
$$

which proves this theorem.

Thus if we know the Laplace transform of a real function, we can use equation 2.9 to obtain the corresponding inverse Laplace transform without performing an integration on the complex plane.

To conclude this section, we give a practical application of the Theorem 2.1 taking into account the formula in equation 2.9 . For this, we consider the Example 1.1 Since $\mathrm{F}(s)=\frac{1}{s^{2}+1}$ is analytic for $\mathcal{R}[s]=\sigma>0$, we can choose any $\sigma>0$ in equation (2.9). In particular, if we choose $\mathcal{R}[s]=\sigma=1$, when we substitute $s=1+i \tau$ into $\mathrm{F}(s)$, we have

$$
\mathrm{F}(1+i \tau)=\frac{1}{(1+i \tau)^{2}+1}=\frac{2-\tau^{2}}{4+\tau^{4}}-i \frac{2 \tau}{4+\tau^{4}}
$$

Separating the real and imaginary parts of $\mathrm{F}(s)$ and substituting equation 2.16 into equation 2.9 , we obtain

$$
f(t)=\frac{e^{t}}{\pi} \int_{0}^{\infty} \frac{\left(2-\tau^{2}\right) \cos (t \tau)+2 \tau \sin (t \tau)}{4+\tau^{4}} \mathrm{~d} \tau, \quad(t>0) .
$$

We performing the integral in equation 2.17) using Mathematica software and we obtain the following result

$$
f(t)=\frac{e^{t}}{\pi} \cdot \pi e^{-t} \sin t=\sin t
$$


Note that the sine function does not satisfy the hypotheses of Theorem 2.1, which suggests a theorem with weaker hypotheses.

Remark. From equation 2.9 , for $t>0$, we can write

$$
\frac{\pi}{2}\left[e^{-\sigma t} f(t)+e^{\sigma t} f(-t)\right]=\int_{0}^{\infty} \mathcal{R}[\mathrm{F}(\sigma+i \tau)] \cos (t \tau) \mathrm{d} \tau .
$$

The function $f$ is such that $f(t)=0$ for $t<0$. Since $t>0$, equation (2.18) yields

$$
f(t)=\frac{2 e^{\sigma t}}{\pi} \int_{0}^{\infty} \mathcal{R}[\mathrm{F}(\sigma+i \tau)] \cos (t \tau) \mathrm{d} \tau .
$$

In a similar way,

$$
f(t)=-\frac{2 e^{\sigma t}}{\pi} \int_{0}^{\infty} \mathcal{I}[\mathrm{F}(\sigma+i \tau)] \sin (t \tau) \mathrm{d} \tau .
$$

Namely, there are three possible cases to find the inverse Laplace transform of a function $\mathrm{F}(s)$, they are given by equations $2.9,2.19$ and 2.20 .

We conclude that the evaluation of the inverse Laplace transform using Theorem 2.1 provides the function $f(t)$ by calculating an integral on the positive real axis. This integral, sometimes can only be determined using some mathematical software. It must be noted, however, that the use of this theorem allows us to express some functions by means of integrals on the positive real axis, as we shall see in the next section.

\section{Results}

Considering the same particular example presented previously in Section 1 (Example 1.1), the equation 2.17) can be rewritten, for $t>0$, in the form

$$
\sin t=\frac{e^{t}}{\pi} \int_{0}^{\infty} \frac{\left(2-\tau^{2}\right) \cos (t \tau)+2 \tau \sin (t \tau)}{4+\tau^{4}} \mathrm{~d} \tau .
$$

In particular, if $t=\frac{\pi}{2}$ in equation 3.21 , we have

$$
\pi e^{-\frac{\pi}{2}}=\int_{0}^{\infty} \frac{\left(2-\tau^{2}\right) \cos \left(\frac{\pi \tau}{2}\right)+2 \tau \sin \left(\frac{\pi \tau}{2}\right)}{4+\tau^{4}} \mathrm{~d} \tau .
$$

Considering the same example, by equation (2.16), we can write the expressions

$$
\mathcal{R}[\mathrm{F}(1+i \tau)]=\frac{2-\tau^{2}}{4+\tau^{4}} \quad \text { and } \quad \mathcal{I}[\mathrm{F}(1+i \tau)]=-\frac{2 \tau}{4+\tau^{4}} .
$$

Substituting $\mathcal{R}[\mathrm{F}(1+i \tau)]$ given by equation 3.23 into equation 2.14 , we have

$$
\frac{2-\tau^{2}}{4+\tau^{4}}=\int_{0}^{\infty} e^{-t} \sin t \cos (t \tau) \mathrm{d} t, \text { for all } \tau \in \mathbb{R},
$$

and into equation 2.15 ,

$$
\frac{2 \tau}{4+\tau^{4}}=\int_{0}^{\infty} e^{-t} \sin t \sin (t \tau) \mathrm{d} t, \text { for all } \tau \in \mathbb{R} .
$$


In particular, if $\tau=0$ in equation 3.24 and $\tau=1$ in equation 3.25, we have

$$
\frac{1}{2}=\int_{0}^{\infty} e^{-t} \sin t \mathrm{~d} t
$$

and

$$
\frac{2}{5}=\int_{0}^{\infty} e^{-t} \sin ^{2} t \mathrm{~d} t
$$

respectively.

On the other hand, it is possible to obtain a more simple integral representation for the sine function than the one in equation (3.21). In fact, if we use equation (2.19), then we can express

$$
\sin t=\frac{2 e^{t}}{\pi} \int_{0}^{\infty} \frac{\left(2-\tau^{2}\right) \cos (t \tau)}{4+\tau^{4}} \mathrm{~d} \tau, \quad \text { for } t>0 .
$$

\section{Concluding remarks}

In this paper we studied two ways to evaluate an inverse Laplace transform. One of them by means of the residue theorem using the complex plane and another without the aid of contour integration. We justify the importance of the second way that lies in the fact that it allows us to find integral representations on the positive real axis for some functions. Furthermore, we believe that it can be presented to graduate students, in many different disciplines in the mathematical, as an additional motivation.

\section{References}

[1] DEAKIN, M. A. B. Motivating the Laplace transform. International Journal of Mathematical Education in Science and Technology, v. 12, n. 4, 1981, p. 415-418. http://doi.org/10.1080/0020739810120407

[2] WIDDER, D. V. Advanced calculus. 2nd ed., New York: Prentice-Hall, 1947.

[3] OBERHETTINGER, F.; BADII, L. Tables of Laplace transforms. New York: Springer-Verlag, 1973.

[4] BELLMAN, R. E.; ROTH, R. S. The Laplace transform. Singapore: World Scientific, 1984.

[5] LUNDBERG, K. H.; MILLER, H. R.; TRUMPER, D. L. Initial conditions, generalized functions and the Laplace transform. Control Systems IEEE, v. 27, 2007, p. 22-35. http://ieeexplore.ieee.org/stamp/stamp.jsp?arnumber $=4064845$

[6] AHLFORS, L. V. Complex analysis: an introduction to the theory of analytic functions of one complex variable. Singapore: World Scientific, 1984.

[7] CAPELAS DE OLIVEIRA, E.; RODRIGUES JR., W. A. Analytical functions with applications (in Portuguese). São Paulo: Editora Livraria da Física, 2006. 
[8] BERBERAN-SANTOS, M. N. Analytical inversion of the Laplace transform without contour integration: application to luminescence decay laws and other relaxation functions. Journal of Mathematical Chemistry, v. 38, n. 2, 2005, p. 165-173. http://doi.org/10.1007/s10910-005-49613

[9] CONThARTEZE GRIGOLETTO, E.; CAPELAS DE OLIVEIRA, E. Is a definition always useful to calculate something? International Journal of Mathematical Education in Science and Technology, v. 44, n. 5, 2013, p. 761-765. http://doi.org/10.1080/0020739X.2012.756554

[10] FIGUEIREDO, D. G. Análise de Fourier e equações diferenciais parciais. Coleo Euclides, Rio de Janeiro: IMPA, CNPq, 1986.

[11] IÓRIO, V. EDP: um curso de graduação, Rio de Janeiro: IMPA, CNPq, 2010.

[12] KNOPP, K. Theory of functions (Parts I and II). New York: Dover, 1996. 